\title{
Developing Applications with Networking Capabilities via End-to-End SDN (DANCES)
}

\author{
Victor Hazlewood $^{1}$, Kathy Benninger ${ }^{2}$, Greg Peterson ${ }^{1}$, Jason Charcalla ${ }^{1}$, Benny Sparks ${ }^{3}$, Jesse Hanley ${ }^{3}$, \\ Andrew Adams ${ }^{2}$, Bryan Learn ${ }^{2}$, Robert Budden ${ }^{2}$, Derek Simmel ${ }^{2}$, Joseph Lappa ${ }^{4}$, Jared Yanovich ${ }^{5}$ \\ ${ }^{1}$ National Institute for Computational Sciences, University of Tennessee \{victor, gdp, jcharcalla $\} @$ utk.edu \\ ${ }^{2}$ Pittsburgh Supercomputing Center \{benninger, akadams, blearn, rbudden, dsimmel@psc.edu\} \\ ${ }^{3}$ National Center for Computational Sciences, Oak Ridge National Laboratory \{sparksbm,hanleyja@ornl.gov\} \\ 4 jlappa@gmail.com \\ ${ }^{5}$ Google, Inc. jaredyanovich@gmail.com
}

\begin{abstract}
The Developing Applications with Networking Capabilities via End-to-End SDN (DANCES) project [1] is a collaboration between The University of Tennessee's National Institute for Computational Sciences (UT-NICS), Pittsburgh Supercomputing Center (PSC), Pennsylvania State University (Penn State), the National Center for Supercomputing Applications (NCSA), Texas Advanced Computing Center (TACC), Georgia Institute of Technology (Georgia Tech), the Extreme Science and Engineering Discovery Environment (XSEDE), and Internet2 to investigate and develop the ability to add network bandwidth scheduling via softwaredefined networking (SDN) programmability to selected cyberinfrastructure services and applications. DANCES, funded by the National Science Foundation's Campus Cyberinfrastructure Network Infrastructure and Engineering (CC-NIE) program award numbers 1341005, 1340953, and 1340981, has field tested five vendor network devices in order to determine which implements the DANCES requirements of the OpenFlow 1.3 standard to provide the network reservation and rate-limiting capability desired to implement the goals of DANCES. Another key device selection criterion was sufficient packet buffering to handle wide area network flows without excessive packet loss. After selection of the network device a test environment was setup between UT-NICS and PSC to perform SDN tests in a simulated supercomputer center compute and data transfer resource environment. This paper describes the DANCES project, the DANCES OpenFlow 1.3 specification requirements, the determination and acquiring of a sufficient OpenFlow 1.3 network device, the provisioning of a test environment, and the test plan and results obtained so far by the DANCES team.
\end{abstract}

\section{CCS Concepts}

- Networks---Network performance evaluation;500

Permission to make digital or hard copies of all or part of this work for personal or classroom use is granted without fee provided that copies are not made or distributed for profit or commercial advantage and that copies bear this notice and the full citation on the first page. Copyrights for components of this work owned by others than the author(s) must be honored. Abstracting with credit is permitted. To copy otherwise, or republish, to post on servers or to redistribute to lists, requires prior specific permission and/or a fee. Request permissions from Permissions@acm.org.

XSEDE16, July 17 - 21, 2016, Miami, FL, USA.

Copyright is held by the owner/author(s). Publication rights licensed to ACM.

ACM 978-1-4503-4755-6/16/07 ..\$15.00

DOI: http://dx.doi.org/10.1145/2949550.2949557

\section{Keywords}

OpenFlow;Software-Defined Networking; SDN; Network Performance; Network Quality of Service; QOS; Data transfer; Internet2; XSEDE; network bandwidth reservation; network scheduling.

\section{BACKGROUND}

Since 2011, XSEDE [2] has been working to integrate NSF's supercomputers, mass storage, data collections, and network resources into a seamless computing environment for scientific and engineering research. An integral part of XSEDE's mission is to enhance accessibility and facilitate the use of these resources by the scientific research community. While XSEDE and its TeraGrid predecessor have been advancing the capabilities of NSF's scientific supercomputing research cyberinfrastructure, the NSFsupported Global Environment for Network Innovation (GENI) [3] has been leading advances in network configurability and control. Support of wide area software-defined networking (SDN) capable networks was pioneered by GENI with the 2010 installation of OpenFlow [4] switches on National LambdaRail (NLR) and Internet 2 backbone paths. In this early deployment, use of an OpenFlow path on either NLR or Internet 2 required detailed planning and coordination between sites. Each participating campus needed to be added individually with manual VLAN provisioning hop-by-hop along the path from the research lab, through the campus, across a metropolitan area network, and finally connection to one of the OpenFlow backbones. While the procedure was sufficient for early experimentation by closely coordinated collaborators, access to OpenFlow control was not readily available on demand.

Since 2010, interest in SDN technology has grown rapidly. Many network hardware vendors are now incorporating and fully supporting SDN in their products. Internet2's 2012 deployment of SDN as the basis for their production research and education (R\&E) network architecture, Advanced Layer2 Services (AL2S), demonstrated a high degree of commitment to, and acceptance of, the technology. AL2S gave Internet 2 connectors a simple web interface to provision their own paths across the WAN between collaborating sites. The AL2S service tracks the provisioned paths and manages the VLAN translation without the detailed hop-byhop coordination and manual configuration that was previously required.

During the first quarter of 2013, the XSEDE network (XSEDEnet) migrated from dedicated optical waves onto Internet2's AL2S infrastructure. A significant benefit of the XSEDE move to AL2S, and an enabling factor of the DANCES project, is that research scientists at AL2S-connected campuses now have a more straightforward and cost-effective way to access XSEDE services 
than with the previous dedicated wave infrastructure. The transition to AL2S also has the advantages of eliminating the $10 \mathrm{GbE}$ bottleneck of the original XSEDE backbone and offering multiple wide area $100 \mathrm{GbE}$ backbone path options between sites, providing redundancy and flexibility. Internet 2 's $100 \mathrm{GbE}$ core infrastructure and aggressive upgrade policy make backbone bandwidth contention unlikely within the wide area AL2S infrastructure.

Though backbone congestion is unlikely due to current trends of overprovisioning R\&E wide area networks, end site infrastructure commonly remains $10 \mathrm{GbE}$ based and is still subject to contention for bandwidth between flows. Network performance monitoring has shown that during peak usage periods, 10GbE XSEDE site and local campus connections do experience congestion, with a negative effect on overall data movement. This was demonstrated by the movement of nearly 1PB of data across XSEDEnet in May and June 2012 when a computing resource was decommissioned, requiring mass migration of user data. With no bandwidth management or Quality of Service (QoS), slowness or blocking of smaller competing flows and unpredictable transfer rates for the bulk transfer occurred. The DANCES network bandwidth scheduling and QoS capability is intended to enable management of network resources to address poor network throughput caused by congestion problems at the end site connection point to the wide area network.

\section{GOALS}

The major project goals of the DANCES project are to perform an assessment of SDN technology, and to build a prototype environment that would add network bandwidth scheduling via SDN programmability to selected cyberinfrastructure applications. The SDN capabilities would be provided by a network device that implements OpenFlow 1.3 including per-flow meters and per-port queueing required by DANCES. The bandwidth control and scheduling capability will be used to mitigate congestion-induced throughput problems on end site networks. The prototype environment setup has been done in two phases. The first phase was done by setting up and testing in a virtual environment and the second phase was done by creating a WAN test environment.

The cyberinfrastructure applications chosen for investigation and potential performance enhancement by DANCES include (1) SCP and GridFTP data transfers and (2) wide area distributed file system data transfers initiated by, and integrated with, job scheduling and resource management. In addition, SLASH2 [5], the wide area distributed file system targeted for DANCES integration, natively supports scheduled remote file replication. Coupling the SLASH2 native file transfer scheduling with the network bandwidth scheduling capability of DANCES increases the efficiency of both network and file system resources.

The project set out to deploy a prototype environment to extend the TORQUE/Moab scheduling and management currently in use at several XSEDE supercomputing sites to support networking as a schedulable resource. The wide area distributed file systems selected for bandwidth scheduling integration were the XSEDEwide File System built on IBM's General Parallel File System (GPFS) and SLASH2 developed at Pittsburgh Supercomputing Center. Since the award of DANCES, XSEDE has decided to no longer support the XSEDE-wide file system based on GPFS and, therefore, DANCES modified its goals to test and prototype using GridFTP, SLASH2 and now SCP as the replacement for GPFS.

Network bandwidth, much like computation time and storage capacity, is a shared resource in high performance computing environments with finite limits. Through SDN technologies, bandwidth can be allocated and usage tracked. With the prototype environment, the DANCES project expected to show how network bandwidth could be considered a true allocable and schedulable resource.

\section{SDN SWITCH AND CONTROLLER REQUIREMENTS AND EVALUATION}

Software Defined Networking has become broadly used terminology, including open and vendor proprietary protocols for network device control communication. Early in the project, the DANCES team chose OpenFlow as the switch control protocol because it was the most widely deployed and supported, as well as being an open standard.

The OpenFlow specification [6] from the Open Networking Foundation [7] has evolved significantly since version 1.0.0 in 2009 and describes the required and optional functions of an OpenFlow compliant switch and the OpenFlow protocol used to manage an OpenFlow switch from a remote controller [8]. In an SDN architecture the control and data plane components of the network are separated. The OpenFlow protocol specification provides a standard interface design for a switch and a protocol for a controller for unlocking the flexibility, scalability and programmability of a network employing an SDN architecture [9]. The need for and value of SDN in the enterprise and in research is well documented, but it is worth mentioning that all OpenFlow switches are not created equal. The current OpenFlow specification released on 26 March 2015 is OpenFlow Switch Specification Version 1.5.1. In this specification and all versions prior to version 1.5.1 the specification indicates a number of switch functions and features of which many are labeled as optional. This has led the DANCES team to the observation that a vendor can identify a switch as being specification version 1.3.0 compliant but have a number of functions and features in the specification not implemented in the switch. The DANCES team identified the version 1.3.0 specification as the minimum target for switch selection in order to provide for complex quality of service frameworks (e.g. rate limiting) that could be provided by implementation of the specification's per-flow meters in section 5.7 and per-port queues in section 5.12 and A.2.2 [10]. Additionally, the per switch port buffer space was of equal importance in switch selection due to high expected bandwidth delay product across the WAN between collaborator sites [11]. The third major factor considered in switch selection was compatibility with an open source OpenFlow controller.

With these three key requirements identified, investigation and testing of several vendors' switches were conducted in the first year of the DANCES project. The team reviewed specifications of Arista, Brocade, Cisco, Dell, Noviflow, and Pica8, spoke with the vendors, and concluded that each of those options did not sufficiently meet all the selection criteria while remaining within the project budget. Five OpenFlow switches were selected for field test. These included an early-release Netronome Network Flow Processing Platform (NFPP-1U), a Juniper EX9204, a Juniper MX80-48T, an HP5920, and a Corsa DP6410, which was eventually selected for the project deployment. The field test verified "traditional" switch device performance criteria such as throughput, fair-sharing of bandwidth among TCP flows, and jumbo frame MTU support in addition to OpenFlow control operation.

Each device tested presented some unique challenges and behaviors. As one of the earliest products to fully support OpenFlow 1.3 and metering, the Netronome NFPP-1U was the first 
switch to be evaluated for the project. The NFPP-1U is designed for LAN applications and therefore includes a relatively small amount of per-port packet buffering. In spite of the small buffering, the team decided to test the NFPP-1U to get experience with a hardware OpenFlow 1.3 implementation. While the NFPP-1U test did provide valuable experience, network throughput testing across the WAN between PSC and UT-NICS confirmed that the switch buffers were too small to support the 10Gbps wide area throughput required for the project.

Both Juniper products that were tested are designed with sufficient per-port buffering to support high bandwidth flows in the WAN. Unfortunately, at the time of the switch evaluation, Juniper did not support OpenFlow 1.3 metering, nor could they estimate when metering support would be available.

Based on specifications and price, the HP5920 was a promising switch candidate. While marketed primarily as a "top of rack", or ToR, switch for LAN applications, the switch is designed with relatively deep packet buffers (3.6GB total). The HP5920 also supports OpenFlow 1.3 with metering as well as traditional Layer 2 / Layer 3 switching and routing services.

Tests with the default HP5920 configuration showed that local flows, with lower round trip times than remote flows traveling across the WAN, would starve the remote flows of bandwidth. Local flows would claim over 9Gbps, leaving only 100's or 10's of Mbps for any remote flows. The DANCES team exchanged several email messages and phone calls with HP engineers in an effort to achieve bandwidth fair-sharing among local and remote flows. One simple fix was enabling use of the HP5920's large packet buffers, which is off by default. With access to the large memory enabled, local flows still tended to receive a larger share of the bandwidth. The sharing of bandwidth varied with several factors: the selected queuing discipline; which switch port a server was connected to (each set of eight ports shares 1GB of buffer memory); and, to a lesser degree, the TCP congestion control protocol used by the end hosts. The team concluded that the HP5920 has the basic functionality, but that achieving an appropriate configuration for the DANCES project will require additional testing and adjustment.

Ultimately, the Corsa DP6410 was selected for deployment in the testbed due to its OpenFlow 1.3.0 feature support including perflow meters and configurable per-port queues and weights, large per-port buffer sizes, bandwidth fair-sharing among local and remote flows, and Corsa's willingness to work with the DANCES team to implement a flow table pipeline specifically to meet the DANCES WAN flow requirements.

Corsa products are labeled by the vendor as "pure OpenFlow data planes", which means that the switch offers only the data plane and a user programmable control plane with no hybrid mode available. In addition to accepting FlowMods (essentially OpenFlow internal routing instructions that modify the switch's flow table) from an OpenFlow controller, the Corsa's flow tables can be modified directly via a command-line interface (CLI). This enabled testing of switch capabilities to begin prior to controller selection and development.

An integral part of any SDN deployment is the controller selection. While OpenFlow was well established as a standard controller-toswitch interface (or "southbound" interface) when the DANCES project began in 2014, the controller-to-application interface (or "northbound" interface) was not standardized. The DANCES SDN controller had to support OpenFlow 1.3 and offer the flexibility and accessibility of open source to enable integration with the XSEDE environment and DANCES control.

Controller investigation for DANCES began with researching and testing the interoperation between Internet2's AL2S OpenFlow environment and application servers at UT-NICS and PSC. The Global Research Network Operations Center (GlobalNOC) at Indiana University provides the Open Exchange Software Suite (OESS) [12], which is the basis for AL2S path provisioning. OESS is used to configure and control dynamic (user-controlled) Layer 2 virtual circuit (VLAN) networks on Internet2's OpenFlow-enabled infrastructure. VLANs can be provisioned either manually through a web-based user interface or programmatically through a web services RESTful API to OESS.

The team at PSC and UT-NICS each provisioned a local VLAN from a test host, through local infrastructure, and to their XSEDEnet edge. Once the path to XSEDEnet was in place at each end, the DANCES team provisioned a circuit between the two endpoints using the OESS web interface then verified connectivity by running iperf3 [13] between the hosts at each end.

The next step was to use the programmatic interface to OESS to create the path between PSC and UT-NICS. The team created an application in C to: (1) provision a WAN VLAN circuit between PSC and UT-NICS using the OESS programmatic interface; (2) run an iperf3 test; and (3) tear down the path when the iperf3 completed. After an authentication issue with the OESS API was resolved, the application worked well and successfully demonstrated the programmatic provisioning capability [14].

The initial approach to OpenFlow controller selection was to download and install the OESS software at PSC to act as the controller for the end site switches along with the OESS instance controlling the WAN. Early in the testing process, when it became clear that OESS only supported OpenFlow 1.0 and there was no committed date for OpenFlow 1.3 support, the DANCES team began looking for alternatives to use at the end sites.

The team selected Ryu [15] and OpenDaylight [16] [17] for evaluation. OpenDaylight is a turnkey solution designed to integrate with production systems. Consequently, it had many features not needed for the DANCES project and was more difficult to customize. In contrast, Ryu is a programming framework, providing the bare-minimum needed for an SDN controller. The open framework architecture made the Ryu controller easier to customize. Additionally, during the first phase of the project, the Ryu framework supported a newer version of the OpenFlow specification than OpenDaylight. While both OpenDaylight and Ryu had the features and functionality required for DANCES, the openness and flexibility of Ryu made it a better choice from a development perspective.

With the SDN hardware and OpenFlow controller selections made, software development and API definition started. As stated previously the OpenFlow specification has been the most commonly used protocol between SDN switches and controllers. Though the protocol is "standard", the controller must be configured to work with the specific vendor's switch. One area of significant difference between vendors' SDN switches is the implementation of flow tables. The DANCES Ryu controller was adapted to work with the multiple flow tables supported by the Corsa switch. 


\section{TEST ENVIRONMENT}

The DANCES team provisioned a WAN testbed with a Corsa DP6410 and server hardware at both PSC and at UT-NICS connected with a VLAN provisioned across XSEDEnet, a private network enabled by Internet2's Advanced Layer 2 Service (AL2S). The DANCES test environment is shown in Figure 1.

Network resources were available at UT-NICS and PSC to provision dedicated test paths within each site so that the DANCES test traffic was not affected by competition with regular production traffic. The testbed included four servers at UT-NICS including: th-c01 running iperf3 and a GridFTP server; th-c02 running iperf3, Torque and Moab resource management and scheduling services, and GridFTP client commands; th-c03 running iperf3 and available for a SLASH2 file system; and th-c04 running iperf3, GridFTP server and a Ryu controller. The testbed included three servers at PSC including: Mambo running iperf3, GridFTP, and HPN-SSHenabled SCP servers [18]; Rumba running iperf3, Torque and Moab, and a SLASH2 file system; and Tango running iperf3, a Ryu controller and a bandwidth management service. The Torque configuration was setup to have a SDN queue. The Torque Configuration is listed on the DANCES website [1] in the Appendix of the DANCES GridFTP Test Results [19].

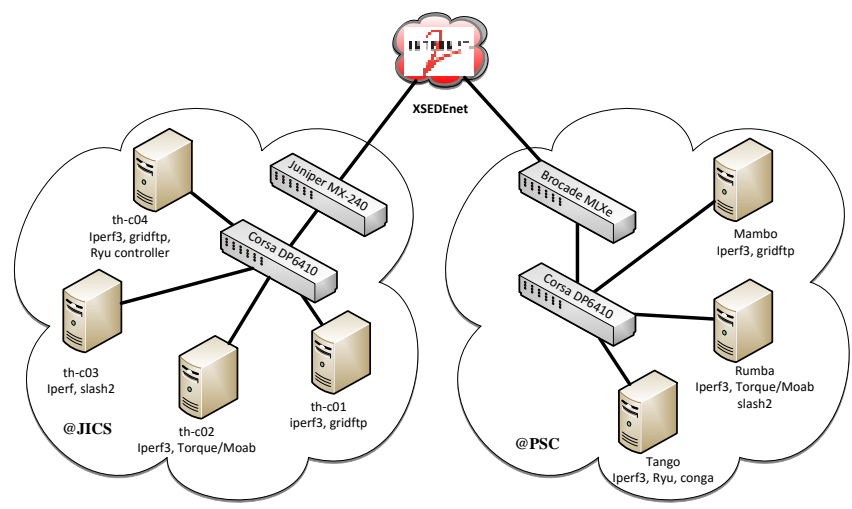

Figure 1: DANCES WAN Test Environment (OVictor Hazlewood

XSEDEnet was used as the network platform since all the collaborators participate in XSEDEnet and it is built upon Internet2's AL2S, which has OpenFlow 1.0 capabilities. DANCES relies on AL2S programmatic VLAN provisioning, but does not require QoS in the WAN due to the 100G backbone relative overprovisioning as compared to the Corsa uplink and server 10G interfaces. OpenFlow 1.3 is also backwards compatible with OpenFlow 1.0.

DANCES coordinated with the XSEDE networking group to implement a separate DANCES point-to-point network in parallel with the production XSEDEnet in order to reduce the possibility of interrupting XSEDEnet production traffic. Once the circuit was established a VLAN was implemented connecting the test environment together. This formed the WAN SDN data plane connectivity for the DANCES project with private IP address space used for the test server interfaces. The DANCES team chose to route all control traffic via the Internet 2 AL3S network using public address space. The SDN control plane can be run in-band, however there are risks inherent in carrying control traffic via dynamically provisioned paths. The Corsa was integrated into the network infrastructure as shown in Figure 1 beyond the Juniper MX240 at UT-NICS and the Brocade MLXe at PSC.

In addition to the WAN testbed, PSC installed a second local test configuration with one Corsa switch and four test servers in PSC's main computer room to easily enable local hands-on access at both ends if required during testing.

\section{TEST PLAN AND RESULTS}

The test environment was setup with the following test capabilities in mind in order to test individual components and to test increasingly larger collections of components in a methodical and modular manner. First, the network path and performance were tested with iperf3 to verify path integrity as well as end host network configuration and TCP tuning. Testing then expanded to incorporate user application and OpenFlow infrastructure-related performance: GridFTP memory-to-memory and GridFTP disk-todisk performance; performance with and without Corsa OpenFlow per-flow meters configured; a resource management capability with Torque and Moab simulating a batch queuing and scheduling capability; installation, configuration and use of a Ryu OpenFlow controller; and submission of a test job using all the previously mentioned services to simulate a data transfer batch job employing network scheduling and perf-flow meters with OpenFlow to rate limit data transfer flows between UT-NICS and PSC across the WAN.

The following made up the test plan to test GridFTP in the DANCES test environment: (1) iperf transfers using max meter (which does not apply marking for bandwidth limiting to a flow); setup with the Corsa CLI; (2) iperf transfers with one transfer using a QOS meter/flow at 1Gbps (CLI setup); (3) GridFTP transfers using max meter (CLI setup); (4) GridFTP transfers with one transfer using a QOS meter/flow at 1Gbps (CLI setup); (5) One or more GridFTP transfer submitted to Torque using max meter (CLI setup); (6) A GridFTP transfer submitted to Torque using a QOS meter/flow at $1 \mathrm{Gpbs}$ (CLI setup); (7) A GridFTP transfer submitted to Torque using a QOS meter/flow at 1 and 5Gpbs (CLI setup) while another GridFTP transfer on max meter is in progress; (8) One or more GridFTP transfer submitted to Torque using max meter using setup with Ryu enabled job start/job end capability; (9) A GridFTP transfer submitted to Torque using a QOS meter/flow at 1 and 5Gbps along with Ryu enabled job start/job end capability while another GridFTP transfer on max meter is in progress simulating a complete supercomputer environment.

The full test results are available on the DANCES website in the GridFTP Throughput Test Results document [19]. The results were very consistent with the use of meters and are summarized in the following.

The baseline iperf3 testing using a max meter on flows between nodes, both locally and over the WAN, revealed some host/path combinations had slower than expected throughput. The performance of the underlying components and servers needed to be optimal to support meaningful testing outcomes of the DANCES infrastructure and new services so these problems had to be identified and corrected before proceeding. Iperf3 throughput between LAN nodes on the same Corsa devices at UT-NICS and at PSC was regularly observed at 9.89 gigabits per second (Gbps), however iperf3 results across the WAN were lower than expected. The team determined that the types of problems encountered were the same as those that frequently occur in non-SDN networks: failing infrastructure components (fiber optic modules and dirty optical fiber ends) and inappropriate end host tuning (TCP buffer and MTU sizes). After these issues were corrected, the iperf3 results improved significantly, iperf3 performance testing between UT-NICS nodes and PSC nodes was observed also at $9.89 \mathrm{Gbps}$ after a few seconds of initial ramp up during the slow start phase of the TCP protocol. 
Iperf3 was also used to check the fairness of bandwidth sharing across multiple parallel flows. A Corsa pre-production switch demonstrated a generally even distribution of bandwidth among local and long round trip time (RTT) flows. The large packet buffers and buffer management of the Corsa effectively maintained the overall fairness. These results are summarized in the Corsa Switch Bandwidth Fair-Share Testing results page [20] on the DANCES website.

After the configuration and performance of the testbed components were optimized and verified, testing and characterization of performance with meters and QoS began. Various combinations of meter rates were used to simulate an environment supporting multiple flows of differing priorities and specified bandwidths along with best-effort traffic flows. This is the type of environment the team expects to encounter in production. The initial production deployment is planned to provide up to a total of 5Gbps of bandwidth for reservation, while $5 \mathrm{Gbps}$ remains available for besteffort traffic.

The following describes the results achieved with the GridFTP test plan. Table 1 shows test results for manual iperf 3 and GridFTP tests with single and multiple flows and max meter or $1 \mathrm{Gbps}$ metering applied.

\begin{tabular}{|c|c|c|}
\hline Test & Meter & Rate achieved \\
\hline Iperf3 & $1 \mathrm{Gbps}$ & $759 \mathrm{Mbps}$ \\
\hline Iperf3 & Max & $8.86 \mathrm{Gbps}$ \\
\hline Iperf3 & $1 \mathrm{Gbps}$ & $724 \mathrm{Mbps}$ \\
\hline Iperf3 + Iperf3 & $1 \mathrm{Gbps}$ & $\begin{array}{l}641 \mathrm{Mbps} \\
374 \mathrm{Mbps}\end{array}$ \\
\hline $\begin{array}{l}\text { GridFTP mem-to-mem } \\
\text { /dev/zero to /dev/null } \\
5 \text { iterations }\end{array}$ & $\operatorname{Max}$ & $\begin{array}{l}\text { Average 5.61Gbps } \\
\text { Low } 4.76 \mathrm{Gbps} \\
\text { High } 8.44 \mathrm{Gbps}\end{array}$ \\
\hline $\begin{array}{l}\text { GridFTP mem-to-mem } \\
\text { /dev/zero to /dev/null } \\
5 \text { iterations }\end{array}$ & $1 \mathrm{Gbps}$ & $\begin{array}{l}\text { Average } 830 \mathrm{Mbps} \\
\text { Low } 520 \mathrm{Mbps} \\
\text { High } 950 \mathrm{Mbps}\end{array}$ \\
\hline $\begin{array}{l}\text { Two simultaneous } \\
\text { GridFTP mem-to-mem } \\
\text { /dev/zero to /dev/null }\end{array}$ & $\begin{array}{l}\text { Max } \\
1 \mathrm{Gbps}\end{array}$ & $\begin{array}{l}6.27 \mathrm{Gbps} \\
840 \mathrm{Mbps}\end{array}$ \\
\hline
\end{tabular}

Table 1. Iperf3 and GridFTP Manual Test Results

GridFTP performance was then tested via a Torque batch job. Table 2 shows these test results with single and multiple flows and max meter or $1 \mathrm{Gbps}$ metering applied.

\begin{tabular}{|l|l|l|}
\hline Test & Meter & Rate achieved \\
\hline $\begin{array}{l}\text { GridFTP mem-to-mem } \\
\text { /dev/zero to /dev/null } \\
5 \text { iterations }\end{array}$ & Max & $\begin{array}{l}\text { Average 4.32 Gbps } \\
\text { Low 3.48 Gbps } \\
\text { High 4.94 Gbps }\end{array}$ \\
\hline $\begin{array}{l}\text { GridFTP mem-to-mem } \\
\text { /dev/zero to /dev/null } \\
5 \text { iterations }\end{array}$ & 1 Gbps & $\begin{array}{l}\text { Average 920 Mbps } \\
\text { Low 860 Mbps } \\
\text { High 950 Mbps }\end{array}$ \\
\hline $\begin{array}{l}\text { Two simultaneous } \\
\text { GridFTP mem-to-mem } \\
\text { /dev/zero to /dev/null }\end{array}$ & Max & $\begin{array}{l}8.15 \mathrm{Gbps} \\
690 \mathrm{Mbps}\end{array}$ \\
\hline $\begin{array}{l}\text { Two simultaneous } \\
\text { GridFTP mem-to-mem } \\
\text { /dev/zero to /dev/null }\end{array}$ & Max & $3.76 \mathrm{Gbps}$ \\
\hline
\end{tabular}

Table 2. GridFTP via Torque Test Results

As can be seen in the throughput results, the actual throughput achieved on the metered flows was less than, but typically at least $70 \%$ of the metered value. A test was also performed where the meter was changed within the job by changing the meter with an
Ryu command implemented with curl just before the GridFTP transfer. This test was successful and worked well.

\section{ACCOMPLISHMENTS AND CONCLUSIONS}

The DANCES project results to date include identification of a network switch, the Corsa DP6410, which demonstrated support for complex quality of service capabilities in the data center and across the WAN. DANCES results show that the OpenFlow 1.3.0 capable flow table pipeline and other features in the Corsa DP6410 provide the capabilities and flexibility needed to support the design goals of DANCES. DANCES configured and tested a prototype supercomputer environment using the Corsa device and showed significant results that network quality of service and network ratelimiting is not only possible in the WAN but can work rather well when integrated into the cyberinfrastructure. Testing showed the power and flexibility of SDN technology. This included the ability to create static flows upon controller initialization and dynamically adding flows to the prototype network. Once this capability is made known to professionals who design and maintain cyberinfrastructure, then infrastructure changes can be made to support these types of rate limiting and scheduled bandwidth reservation capabilities on data transfer resources like those available at XSEDE Service Provider sites and other cyberinfrastructure locations.

With the knowledge gained to date by the implementation of the DANCES project, the DANCES collaborators including XSEDE and XSEDE SPs have a strong foundation for implementing scheduling of network resources utilizing OpenFlow 1.3 feature sets with a Corsa DP6410 device to provide network performance reservation and network quality of service rate limiting on specific data transfer flows within the datacenter and across the WAN. Armed with this information network engineers and system administrators can configure environments that can significantly impact overall researcher workflows including the network data flows between data transfer resources at separate organizations across the WAN. The test environment at UT-NICS will remain active through 2016 for use by the PSC DANCES project and to demonstrate this capability with other organizations who want to participate in scheduling of network resources and network bandwidth reservation capabilities between UT-NICS and data transfer resources at their organization.

\section{FUTURE WORK}

As alluded to in section 4, DANCES includes a bandwidth management component, the Centralized OpenFlow and Network Governing Authority (CONGA) [21], which was developed for the project. The primary function of CONGA is to receive bandwidth requests from the resource manager/scheduler system or the SLASH2 file system and determine if the request can be fulfilled. The decision to accept or reject is based on CONGA's (1) tracking of dedicated and available bandwidth at each site and (2) a user database query to verify that a user or project team member requesting reserved bandwidth has authorization and a sufficient allocation to carry out the request. The XSEDE accounting group created a prototype user authorization database, based on the XSEDE Central Database (XDCDB) format, with a RESTful API for the DANCES team to use to test the interface with CONGA. If the bandwidth is to be allocated, CONGA will signal the OpenFlow controller to proceed with the provisioning.

Figure 2 shows the hardware and software components of the endto-end DANCES infrastructure. The next part of the test plan is to use the control functionality of CONGA with the Ryu controller to 
authorize and set up the user requested bandwidth for end-to-end data transfers between the file servers. Initial testing will be done with a scripted iperf3, followed by GridFTP, scp, and SLASH2 transfers submitted to Torque/Moab (or SLURM).

The basic operational model for the DANCES system with a userinitiated request for bandwidth would proceed as follows:

1. User submits a job via Torque/Moab

2. The job submission will include a Torque prologue script to initiate a bandwidth request to CONGA.

3. CONGA will check the requestor's authorization and the bandwidth availability

4. If both criteria are met, CONGA will signal the DANCES OpenFlow controller to provision the path with the requested bandwidth (If one or both of the criteria are not met, the transfer will proceed with best-effort bandwidth)

5. Once the path has been set up, the job / transfer will proceed

6. A Torque epilogue script will issue a request to remove the provisioning when the transfer has completed

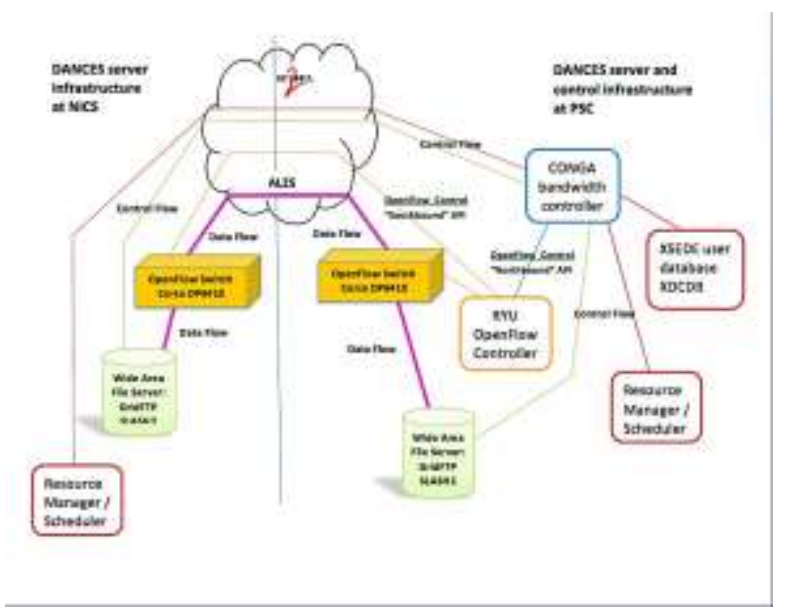

Figure 2. DANCES Functional Diagram @Kathy Benninger

After the operation of CONGA, the API [22], and the control functionality have been verified, the team will test throughput with mixed network traffic. This will build on the GridFTP test effort and include bandwidth-protected and best-effort flows. The team plans to use $1 \mathrm{Gbps}$ as the bandwidth request granularity and support increments in size up to an aggregated total of $5 \mathrm{Gbps}$ (e.g., three reservations at $1 \mathrm{Gbps}+2 \mathrm{Gbps}+2 \mathrm{Gbps}$, two reservations at $2 \mathrm{Gbps}+3 \mathrm{Gbps}$, or one reservation at $5 \mathrm{Gbps}$ would be acceptable), reserving the remaining $5 \mathrm{Gbps}$ of the $10 \mathrm{Gbps}$ network connection for best-effort, non-scheduled flows. Along with the throughput testing, the team will characterize the impact on throughput of adjustments in queue size and weighting within the Corsa switch. The intended outcome is to develop a better understanding of how the switch settings impact performance and recommended settings to achieve optimal throughput for the DANCES managed traffic.

In parallel with the CONGA functional verification, SLASH2's scheduled replication service will be enhanced with the capability to issue requests directly to CONGA for bandwidth reservation. Testing of HPN-SSH-enabled SCP within a Torque/Moab managed workflow will be undertaken. The DANCES team also plans to pursue additional deployment opportunities in 2016.

The current DANCES architecture uses one Ryu controller for all the switches to simplify the bandwidth management logic. However, the team understands the value of local control and plans to investigate the options for supporting and coordinating multiple controllers in the future.

\section{ACKNOWLEDGEMENTS}

This work was supported by the National Science Foundation (NSF) Campus Cyberinfrastructure - Network Infrastructure and Engineering (CC-NIE) program award numbers 1341005, 1340953, and 1340981. Any opinions, findings and conclusions or recommendations expressed in this material are those of the author(s) and do not necessarily reflect the views of the NSF.

\section{REFERENCES}

[1] DANCES project, accessed 16 April 2016, https://www.dances-sdn.org/.

[2] Towns, John, Timothy Cockerill, Maytal Dahan, Ian Foster, Kelly Gaither, Andrew Grimshaw, Victor Hazlewood et al. "XSEDE: accelerating scientific discovery." Computing in Science \& Engineering 16, no. 5 (2014): 62-74.

[3] Berman, Mark, Jeffrey S. Chase, Lawrence Landweber, Akihiro Nakao, Max Ott, Dipankar Raychaudhuri, Robert Ricci, and Ivan Seskar. "GENI: A federated testbed for innovative network experiments." Computer Networks 61 (2014): 5-23.

[4] McKeown, Nick, Tom Anderson, Hari Balakrishnan, Guru Parulkar, Larry Peterson, Jennifer Rexford, Scott Shenker, and Jonathan Turner. "OpenFlow: enabling innovation in campus networks." ACM SIGCOMM Computer Communication Review 38, no. 2 (2008): 69-74.

[5] Pittsburgh Supercomputing Center, "SLASH2," accessed 21 April 2016, https://github.com/pscedu/slash2

[6] Open Networking Foundation, "OpenFlow switch specification," accessed 17 April 2016, https://www.opennetworking.org/technicalcommunities/areas/specification.

[7] Open Networking Foundation, accessed 17 April 2016, https://www.opennetworking.org/.

[8] Open Networking Foundation, "OpenFlow 1.5.1 Specification,” 26 March 2015, accessed 17 April 2016, https://www.opennetworking.org/sdn-resources/technicallibrary.

[9] Open Networking Foundation. "Software-Defined Networking: The New Norm for Networks." ONF White Paper (2012).

[10] Open Networking Foundation, "OpenFlow 1.3.0 Specification," 25 June 2012, accessed 17 April 2016, https://www.opennetworking.org/sdn-resources/technicallibrary.

[11] ESnet. "Router/Switch Buffer Size Issues." accessed 25 April 2016, https://fasterdata.es.net/network-tuning/routerswitch-buffer-size-issues/.

[12] Indiana University Global Research Network Operations Center. "OESS: Open Exchange Software Suite." accessed 25 April 2016, http://globalnoc.iu.edu/sdn/oess.html.

[13] iperf3: A TCP, UDP, and SCTP network bandwidth measurement tool, accessed 29 April 2016, https://github.com/esnet/iperf.

[14] DANCES OESS User Agent. accessed 25 April 2016, https://github.com/pscedu/oess-client.

[15] Ryu, accessed 25 April 2016, https://osrg.github.io/ryu/. 
[16] Azodolmolky, Siamak. Software Defined Networking with OpenFlow. Packt Publishing Ltd, 2013.

[17] Linux Foundation. “OpenDaylight,", accessed 21 April 2016, https://www.opendaylight.org/.

[18] HPN-SSH, https://www.psc.edu/index.php/hpn-ssh, accessed 29 April 2016.

[19] DANCES Test Results: GridFTP Throughput, accessed 9 June 2016, https://www.dances-sdn.org/index.php/projectdocumentation/test-results.
[20] DANCES Test Results: Corsa Switch Bandwidth Fair-Share Testing, accessed 9 June 2016, https://www.dancessdn.org/index.php/project-documentation/test-results.

[21] DANCES CONGA, accessed 21 April 2016, https://github.com/pscedu/conga.

[22] DANCES CONGA API, accessed 29 April 2016, https://www.dances-sdn.org/index.php/projectdocumentation/software/conga-api. 